\title{
Spectroscopy of candidate electromagnetic counterparts to gravitational wave sources
}

\author{
Iain A. Steele, Chris M. Copperwheat and Andrzej S. Piascik
}

Astrophysics Research Institute, Liverpool John Moores University, L3 5RF, UK

\begin{abstract}
A programme of worldwide, multi-wavelength electromagnetic follow-up of sources detected by gravitational wave detectors is in place. Following the discovery of GW150914 and GW151226, wide field imaging of their sky localisations identified a number of candidate optical counterparts which were then spectrally classified. The majority of candidates were found to be supernovae at redshift ranges similar to the GW events and were thereby ruled out as a genuine counterpart. Other candidates ruled out include AGN and Solar System objects. Given the GW sources were black hole binary mergers, the lack of an identified electromagnetic counterpart is not surprising. However the observations show that it is possible to organise and execute a campaign that can eliminate the majority of potential counterparts. Finally we note the existence of a "classification gap" with a significant fraction of candidates going unclassified.
\end{abstract}

Keywords. gravitational waves

\section{Introduction}

As part of the effort to discover and characterise astronomical gravitational wave (GW) sources, a worldwide programme of electromagnetic (EM) follow-up has been established (Abbot et al. 2016a). The programme is organised under the auspices of a series of Memoranda of Understanding (MOU) with over 70 groups who have access to observing resources that can participate in the follow-up. The MOU maintains confidentiality until the discoveries are announced. In the first aLIGO/Virgo observing run (O1) a number of candidate optical counterparts were identified by various wide field optical imaging facilities. In this paper we mainly discuss the work carried out by the 2.0 metre robotic Liverpool Telescope (LT) to spectroscopically follow up a number of those candidates. We also draw some broad conclusions about a potential spectroscopic "classification gap". More details of the work presented here can be found in Copperwheat et al. (2016).

\section{Expected Optical Signatures}

It is a reasonable expectation that binary mergers involving one or more neutron stars (NS) should show a transient EM signature due to energetic outflows. An EM signature is less likely for the merger of two black holes $(\mathrm{BH})$. For NS+NS and NS+BH mergers we can anticipate a number of scenarios based on the assumption that the energetic outflow will have a jet configuration:

- If the observer is within the jet opening angle, a "prompt" spectral signature similar to a short GRB (e.g. Berger 2014) might be expected.

- If the observer is outside the jet opening angle, then (infra-)red kilo-nova emission from radioactive decay of heavy elements synthesised in the merger ejecta is predicted (e.g. Li \& Paczyński 1998). Such emission is likely to be delayed with respect to the GW signal. 
Table 1. Classifications of transients candidates followed by the LT in response to event GW151226. Where we obtain a secure supernova classification we provide a redshift, the time $t$ since peak, and the percentage of matching templates in the SNID database which are consistent with the spectrum.

\begin{tabular}{ll} 
Candidate ID & Comments \\
\hline iPTF-15fed & No transient detected to limiting magnitude of $R \sim 19.1$ \\
iPTF-15fel & Supernova Type Ia, $z=0.038, t=+40 \mathrm{~d}, 97.7$ per cent template fit \\
iPTF-15fev & Supernova Type Ia, $z=0.023, t=+50 \mathrm{~d}, 94.7$ per cent template fit \\
iPTF-15ffh & Possible supernova Type Ia, $z=0.061, t=+15 \mathrm{~d}$ \\
iPTF-15ffi & Supernova Type Ia, $z=0.085, t=+3 \mathrm{~d}, 89.1$ per cent template fit \\
iPTF-15ffk & Supernova Type Ia, $z=0.102, t=+5 \mathrm{~d}$ \\
iPTF-15ffm & Supernova Type Ia, $z=0.094, t=+36 \mathrm{~d}$ \\
iPTF-15ffz & Emission lines consistent with AGN at $z \sim 0.07$ \\
iPTF-15fgy & Supernova Type Ia, $z=0.076, t=+20 \mathrm{~d}, 84.7$ per cent template fit \\
iPTF-15fhd & Possible supernova Type Ia, $z=0.091, t=+11 \mathrm{~d}$ \\
iPTF-15fhl & Possible supernova Type Ib, $z=0.043, t=+18 \mathrm{~d}$ \\
iPTF-15fhp & Possible supernova Type Ic, $z=0.129, t=+1 \mathrm{~d}$ \\
iPTF-15fhq & Narrow emission lines, consistent with AGN at $z=0.043$ \\
iPTF-15fib & Slow moving asteroid \\
LSQ15bvw & No transient detected to limiting magnitude R 19.5 \\
MASTER OTJ020906 & No transient detected to limiting magnitude R 20 \\
UGC 1410 transient & No transient detected. ID'd as minor planet 2606 Odessa \\
\hline
\end{tabular}

- If the jet Lorentz factor is low, we may find a "failed GRB" orphan afterglow which lacks high energy emission but still has an X-ray/optical/radio signature (Lamb \& Kobayashi 2016).

\section{Methodology}

The aLIGO/Virgo consortium have put in place a number of mechanisms for communication of information about GW events and the subsequent follow-up activity between the MOU partners. These include GCN Notices (machine readable information packets that can be rapidly distributed), GCN Circulars (human readable information that is distributed by email), and a machine and human read/write database system (GraceDB) that distributes information on burst times, localisations (sky maps) and false alarm rates. All MOU partners are able to use the GCN and GraceDB systems to provide information regarding their follow-up activities (e.g. search footprints and candidate counterparts) to other MOU partners.

The natural follow-up sequence for the search is to use wide field facilities to identify potential EM counterparts in the area covered by the GW localisation sky map followed by more detailed observations with narrower field instruments. Examples of some of the optical wide field instruments used during O1 include iPTF, PanSTARRS, SkyMapper, VISTA, MASTER, TOROS, TAROR, VST, DECam and Pi of the Sky. At higher energies facilities included Fermi, INTEGRAL, SWIFT. In the radio regime MWA, ASKAP and LOFAR were used. Follow-up of counterparts was carried out by facilities including Keck, PESSTO, UH2.2 and LT (optical spectroscopy) and VLA (radio).

\section{Results}

During O1 three GW triggers were distributed to the MOU partners:

- GW150914 (Abbott et al. 2016b). This comprised a $36_{-4}^{+5}+29_{-4}^{+4} \mathrm{M}_{\odot}$ black hole 


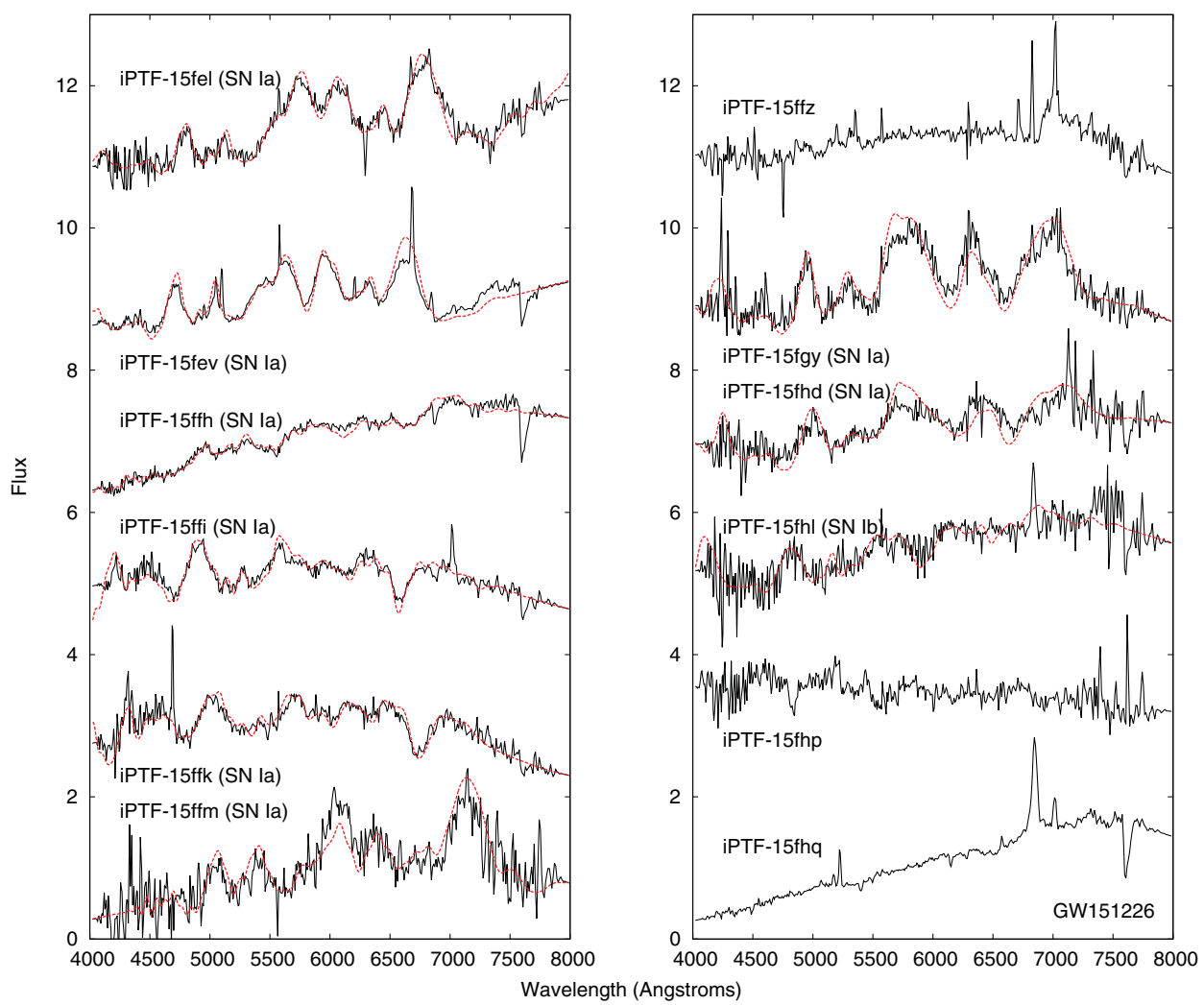

Figure 1. LT spectra obtained during the follow-up of GW151226. We omit candidates for which our observations showed no evidence of a transient. For objects where a supernova identification is obtained we overplot the best template fit.

merger at redshift $z \sim 0.09$. It was detected on 2015 Sept 14 (just before the official start of O1) and an alert issued to the MOU partners on 2015 Sept 16.

- G194575 (LIGO Scientific Collaboration and Virgo 2015). This alert was issued on 2015 Oct 22 but retracted on 2015 Nov 11 when the false alarm rate was recomputed and the event was determined not likely to be a real event.

- GW151226 (Abbott et al. 2016c). This comprised a $14.2_{-3.7}^{+8.3}+7.5_{-2.3}^{+2.3} \mathrm{M}_{\odot}$ black hole merger also at redshift $z \sim 0.09$. It was detected on 2015 Dec 26 and an alert issued on 2015 Dec 27.

For all three of these triggers extensive follow-up was carried out. A summary of the GW150914 follow-up campaign is presented in Abbott et al. (2016d). Figure 1 shows a selection of the spectra obtained by the LT using the low resolution $(R \sim 350)$ SPRAT spectrograph (Piascik et al. 2014) in the follow-up of GW151226. A summary of all of the LT classifications for that event is presented in Table 1. Examining the table shows the majority of candidate counterparts detected are supernovae in the redshift range $z \sim 0.02-0.13$. Some Solar System sources and AGN are also identified, as well as a number of objects where the transient source had faded back into the galaxy background before a spectrum could be obtained. None of the candidates could be associated with GW151226. 


\section{Conclusions}

Given that the $\mathrm{O} 1 \mathrm{GW}$ sources were both $\mathrm{BH}+\mathrm{BH}$ systems (where we do not expect an EM signature) the overall results of the follow-up and classification programme are encouraging. We have shown that a 2 -metre class telescope with a high-throughput, low resolution spectrograph can eliminate many candidate counterparts at redshifts similar to the GW sources. In future aLIGO/Virgo observing runs it is proposed that a distance estimate ("3d sky localisation" - Singer et al. 2016) and "EM-BRIGHT" flag (indicating the possible presence of a neutron star) will be distributed with the alerts. Localisations will also improve as more GW stations come on-line. All of this will help with targeted follow-up of candidates associated with potential host galaxies in the correct redshift range.

For GW151226 a total of 77 candidate optical counterparts were announced via GCN. We note that (over all of the facilities involved):

- 37 of these received a firm spectral classification,

- a further 18 had a more tentative classification based on photometric light curves,

- there were 3 cases where the transient had faded into the host galaxy before spectroscopy could be attempted.

It follows that 19 candidates were not followed up. It is therefore clear that there is a significant danger of a "classification gap" opening up, where potential counterparts will be discovered at a significantly faster rate than can be spectroscopically followed up. While localisation error boxes are anticipated to reduce in size (from the current hundreds of square degrees to tens of square degrees) as more GW stations come on line, it is also anticipated that event numbers will increase by an order of magnitude over the next few years as detector sensitivities improve. The gap is therefore likely to remain a problem. In addition we note that new sources of transients such as LSST (predicted to discover $\sim 10^{6}$ transients/night - LSST Science collaboration et al. 2009) will also be in competition for spectroscopic follow-up time.

To reduce the classification gap we propose the community must (a) start moving to more automated and efficient methods of triggering spectroscopic follow-up of candidates using technologies such as RTML (Hessman 2006) and VOEvent (Williams \& Seaman 2006) and (b) consider the construction of optimized spectroscopic follow-up facilities with large apertures and fast slew speeds (e.g. Liverpool Telescope 2 - Copperwheat et al. 2015).

\section{References}

Abbott, B. P., Abbott, R., Abbott, T. D., et al. 2016a, Living Reviews in Relativity, 19, 1

Abbott, B. P., Abbott, R., Abbott, T. D., et al. 2016b, Phys. Rev. Lett., 116, 061102

Abbott, B. P., Abbott, R., Abbott, T. D., et al. 2016c, Phys. Rev. Lett., 997

Abbott, B. P., Abbott, R., Abbott, T. D., et al. 2016d, ApJ, 826, L13

Berger, E. 2014, ARAA, 52, 43

Copperwheat, C. M., Steele, I. A., Piascik, A. S., et al. 2016, MNRAS, 462, 3528

Copperwheat, C. M., Steele, I. A., Barnsley, R. M., et al. 2015, Experimental Astron., 39, 119

Hessman, F. V., 2006, AN, 327, 751

Lamb, G. P \& Kobayashi, S. 2016, ApJ, 829, 112

Li, L.-X. \& Paczyński, B. 1998, ApJ, 507, L59

LIGO Scientific Collaboration and Virgo 2015a, GRB Coordinates Network, 18442

LSST Science Collaboration, et al., 2009, arXiv:0912.0201

Piascik, A. S., Steele, I. A., Bates, S. D., et al., 2014, Proc. SPIE, 9147, 91478H

Singer, L. P., Chen, H.-Y., Holz, D. E., et al. 2016, ApJ, 829, L15

Williams, R. D. \& Seaman, R., 2006, ASPC, 351, 637 